\title{
Studies of a model mitral valve with three cusps
}

\author{
B. J. Bellhouse, F. H. Bellhouse, and A. J. Gunning \\ From the Department of Engineering Science, Oxford University, and Nuffield Department of Surgery, \\ Radcliffe Infirmary, Oxford
}

Experiments with a model left ventricle and a three-cusp mitral valve are described. The aortic cusp is shown to make larger excursions than the other two cusps, because of an asymmetric vortex filling the left ventricle in diastole. A possible explanation of the late failure of three-cusp mitral fascia-lata mitral valves is proposed.

Late failures of fascia lata mitral valves are reported (Ross, 1972) where the aortic-related cusp remains flexible and functional, but the other two cusps are retracted and tend to fuse across their common commissure. These valves consisted of three equal cusps mounted on a rigid frame, and the reduction in valve orifice diameter appears to be small. Ross (1972) suggests that the abnormal flow pattern in the left ventricle, resulting from the use of the rigid tricuspid valve, may well be responsible for disappointing results with reduced functional efficiency and abnormal contractility on left ventricular angiography. In addition, he suggests that the abnormal and turbulent flow may be responsible for the more recent finding of left ventricular endocardial fibrosis around these prostheses (Roberts and Morrow, 1967).

We have undertaken detailed studies of hydraulic models of the left ventricle and aortic valve (Bellhouse and Bellhouse, 1968b, 1969a) and mitral valves (Bellhouse and Bellhouse, 1969b, Bellhouse, 1972). Our earliest models of mitral valves were tricuspid and were mounted on rigid supporting frames, aligned so that one cusp corresponded to the aorta. These experiments havenot been reported previously, because they were superseded by experiments using a bicuspid mitral valve, which was a better model of the natural mitral valve. They are reported now, because they seem to offer a logical explanation of Ross's clinical findings.

\section{Methods}

A model of the left ventricle was built (Fig. I) consisting of a rigid base made of perspex, incorporating a mitral (A) and an aortic valve (B), and a transparent rubber bag (C) simulating the remainder of the ventricle. The model ventricle was fixed inside a perspex tank (D), Received 5 April 1973. which was filled with water and connected at (E) to a piston pump to actuate the ventricle.

The aortic valve was connected by a pipe (F) to a header tank which overflowed to a lower tank which discharged through a viewer $(\mathrm{G})$ to the mitral valve. The aortic valve had three thin (O.I $\mathrm{mm}$ ) flexible cusps made of silicone rubber reinforced with fabric, and had three sinuses. The mitral valve (Fig. 2a) also had three cusps

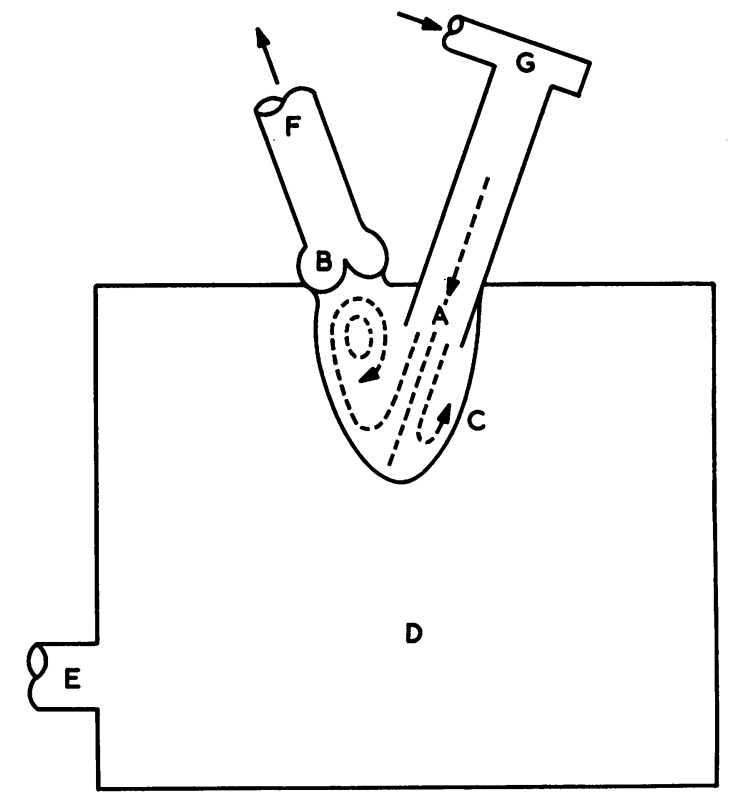

FIG. I Diagram of the model left ventricle, showing the mitral $(A)$ and aortic $(B)$ valves, with a transparent rubber bag $(C)$ simulating the remainder of the ventricle. Water was pumped in and out of the tank $(D)$ by a piston pump connected at $E$ to actuate the ventricle. The ventricle pumped water through a separate circuit, through the aorta $(F)$ to two header tanks back through a viewer $(G)$ into the ventricle. 


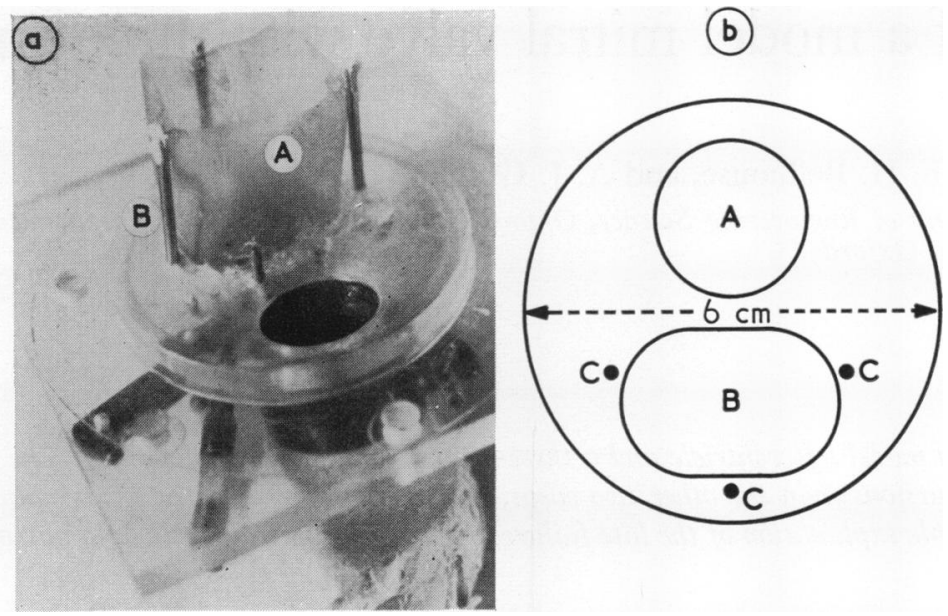

FIG. 2 (a) Photograph of the base of the model left ventricle showing the mitral valve which had three cusps $(A)$ supported at their free margin by three struts $(B)$. (b) Plan of the base of the model ventricle, showing the dimensions of the aortic $(A)$ and mitral $(B)$ rings, and the positions of the supporting struts $(C)$ for the mitral valve.

(A) made of fabric-reinforced silicone rubber. The cusps were supported by three stainless steel struts (B) at three points on the cusp-free margins. The cusps were made from a single sheet made into a divergent cone $83 \mathrm{~mm}$ perimeter at the mitral ring, $100 \mathrm{~mm}$ at the free margin, and $30 \mathrm{~mm}$ in length. The dimensions of the aortic (A) and mitral rings (B) and the positions of the struts (c) supporting the mitral valve are shown in Fig. 2b. The mitral ring was approximately elliptic, with axes of $30 \mathrm{~mm}$ and $20 \mathrm{~mm}$.

The position of the mitral cusps was observed from the atrial side through the viewer (G) shown in Fig. I. The pipe connecting the viewer to the mitral ring was circular and of $25.4 \mathrm{~mm}$ internal diameter. The piston pump actuated a trip-switch, which switched on a light bulb placed in the field of view of a cine-camera. The light and the mitral valve were filmed simultaneously at 50 frames per second. The film was then analysed frame by frame and the area of the mitral cusp orifice was measured as a function of time. (The thermal lag in the bulb was measured and a small correction applied to the results.)

Velocity was measured at the mitral ring with heated thin-film needle-probes (Bellhouse and Bellhouse, 1968a). The probes had outputs linear with velocity, and had a frequency response sufficient to detect turbulence. These probes were traversed across the mitral ring to obtain spatially-averaged flow rates.

The voltage pulse which actuated the timing light was recorded simultaneously with the velocity measurements, which were then correlated with cusp position recorded on cine film.

\section{Experimental results}

Flow patterns in model left ventricle

The ventricle was made to fill and contract with approximately sinusoidal flows (one half for systole and other for diastole), and flow patterns were made visible with solid particles suspended in the water. The mitral valve opened rapidly at the start of diastole, until the cusps were nearly parallel, which was less than their fullest extent. After the valve had opened, the incoming jet struck the apex of the ventricle, then turned back along the ventricle walls to the base; it then changed direction again, to flow behind the cusps towards the apex again, forming a ring vortex (Fig. 3a). The vortex was asymmetrical,
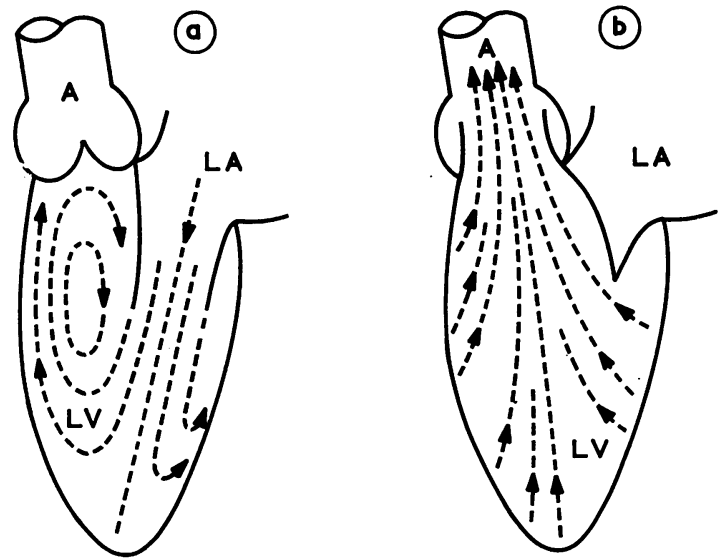

FIG. 3 (a) Flow pattern in the model left ventricle $(L V)$ at mid-diastole. Note the strong asymmetric vortex. The left atrium is marked $L A$, and the aorta $A$. (b) Flow pattern in the model left ventricle at midsystole. 

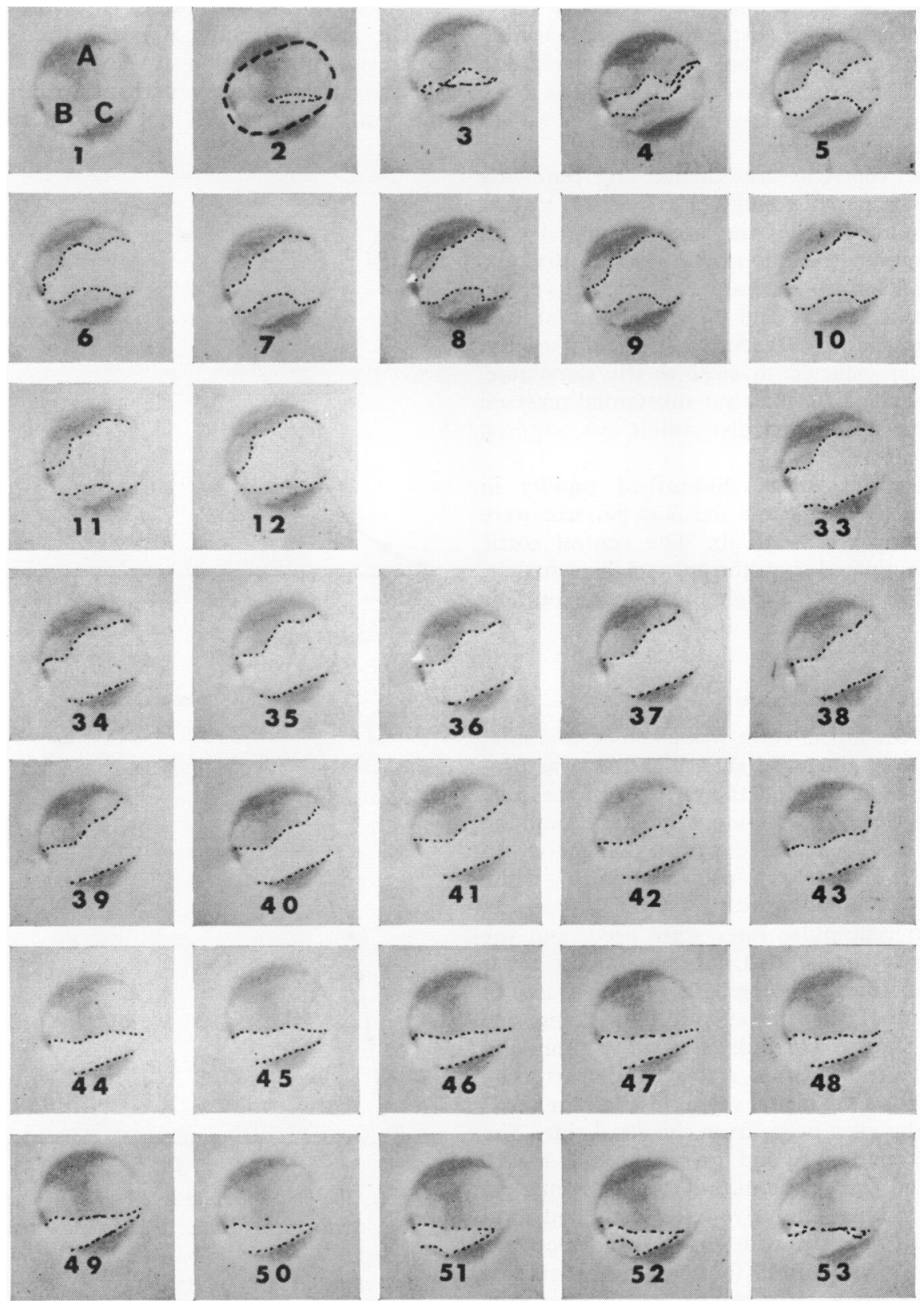

FIG. 4 Successive frames of a cine-film of the three-cusp mitral valve taken at 50 frames a second from the atrial side (pulse rate 29/min). The aortic cusp (marked $A$ in frame $I$ ) is at the top of each frame, and makes significantly greater excursions than the other two cusps $(B, C)$ under the action of the ventricular vortex. The edges of the mitral ring are shown by the broken line in frame 2. Diastole ends at frame 51, when the mitral valve is three-quarters closed. Frames 13 to 32 have been omitted but the complete sequence may be obtained from the authors. 
with its main strength concentrated in the outflow tract, behind the aortic cusp in the mitral valve. After peak diastole, the aortic cusp moved steadily towards closure, and just before ventricular filling was complete, the other two cusps started to close also. Only a small amount of reversed flow was required to seal the valve.

When the ventricle was dilated, by removing water from the perspex tank (D in Fig. I), so that the end-systolic volume was large compared with the stroke volume, the kinetic energy of the diastolic jet was insufficient to generate a ventricular vortex. Keeping stroke volume and pulse rate the same as before, the mitral valve performed quite differently. All these cusps started to close at the same rate, towards the end of diastole, but substantial reversed flow at the onset of ventricular systole was required to seal the valve.

In systole, the vortex diminished rapidly in strength, and by midsystole the flow patterns were like those shown in Fig. 3b. The central aortic streamline originated from the apex of the ventricle, and the velocities were high only in the outflow tract.

\section{Cine film of mitral valve}

Cine film of the mitral valve was taken at 50 frames per second from the atrial side. The film was projected frame by frame and the outlines of the cusp free margins were traced on to white paper, and the area enclosed was measured with a planimeter. In addition these frames were projected on to a white screen and photographed with a $35 \mathrm{~mm}$ still camera.

Three different pulse rates were used, and the results were so similar that only the middle set is presented. In Fig. 4 photographs of each frame of the cine film of the mitral valve, spanning one diastole, are shown. The pulse rate was $29 / \mathrm{min}$, and the successive frames were $1 / 50$ second apart. The first frame shows the mitral valve closed at the onset of diastole, the view being from the atrial side. The aortic cusp is marked A, and the other two B and C. On the second frame, the mitral annulus is marked with a broken line. The valve opens uniformly and quite rapidly, and is wide open by frame 14 . This position persists until frame 33, when gradual closure of the aortic cusp begins. By frame 44 the aortic cusp covers half the mitral annulus. The valve is nearly three-quarters closed by the end of diastole (frame 5I). The onset of ventricular systole completes valve closure in only 3 more frames. A significant feature of these photographs is that this tricuspid valve appears to be bicuspid.

The results are presented differently in Fig. 5, where the velocity at the mitral ring is plotted as a function of time in Fig. 5a, and the corresponding area of the mitral cusp orifice $A$ (divided by the area of the mitral annulus $A_{0}$ ) is plotted in Fig. 5b There was no variation in velocity with position in the mitral ring, except perhaps within $0.5 \mathrm{~mm}$ of the wall, which could not be traversed with the probe.
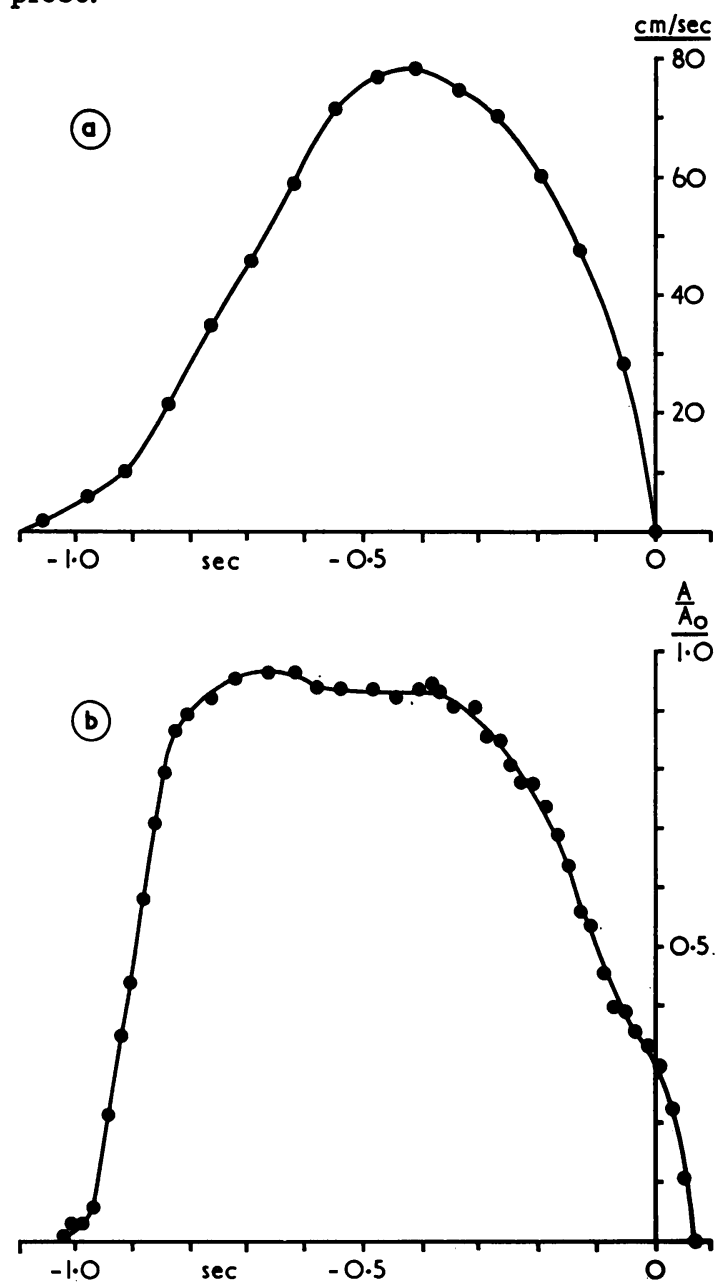

FIG. 5 (a) Measurements of velocity at the mitral ring plotted against time. (b) Measurements of mitral cusp orifice area $(\mathrm{A})$, divided by $\left(\mathrm{A}_{0}\right)$, the area of the mitral annulus, are plotted against time. The pulse rate was 29/min.

Thus the point of zero velocity coincides with zero flow. We deduce that the model mitral valve was 70 per cent closed at the end of diastole.

\section{Discussion}

The difference in cusp movements of the three-cusp mitral valve is due to the asymmetric vortex formed 
in the left ventricle during diastole, which acts preferentially on the aortic cusp and tends to close it. The vortex is not the only determinant of cusp movements; in addition, accelerating flow through the mitral annulus tends to open the valve, decelerating flow to close it. There is also a Venturi effect which tends to hold the cusps parallel. (If the cusps converge, fluid velocity level with the face margins is increased, and the pressure is reduced. This pressure reduction is transmitted to the entire left ventricle, and the cusps tend to open.) These three effects apply to bicuspid and three-cusp mitral valves, and are reported in detail elsewhere (Bellhouse, 1972). They account, too, for the response of the mitral valve to atrial systole, with a partial reopening at the onset of atrial systole (accelerating flow through the mitral annulus) and a resumption of closure later in atrial systole (decelerating flow).

In our models the flow patterns in the left ventricle were similar whether the mitral valve had two or three cusps, and were insensitive to small changes in ventricular geometry. Indeed the three-cusp mitral valve appeared to be bicuspid.

If fascia lata three-cusp valves function in a similar way in the mitral position in man, then this could be an explanation of their mode of failure, with the retraction and fusion of the two 'posterior' cusps.

One obvious measure is to replace the three-cusp frame-mounted valve with a similar two-cusp valve. It is not clear, however, that the haemodynamic benefits resulting from this design change will be significant, when compared with the disadvantages of a rigid supporting-frame for the fascia lata valve.

\section{Conclusion}

Experiments with a model left ventricle and a threecusp mitral valve showed that the mitral valve opened wide early in diastole, and was almost threequarters closed before the onset of ventricular sys- tole. Only a small amount of reversed flow was required to seal the valve. The aortic cusp made much greater excursions than the other two cusps which moved as one. The differences in movements were shown to be due to a vortex, generated in the ventricle in diastole, with its main strength concentrated in the outflow tract, behind the aortic cusp. When the vortex was prevented from forming, by dilating the ventricle, the mitral valve closed late, with all three cusps moving together, and with more regurgitation.

Experiments with model tricuspid and bicuspid valves were compared, and the results shown to be similar. It was suggested that three-cusp fascia lata valves placed in the mitral position may function in the same way, and that the late failures of these valves reported by Ross (1972) may be due to the bicuspid mode of operation of the three-cusp prosthesis.

\section{References}

Bellhouse, B. J. (1972). Fluid mechanics of a model mitral valve and left ventricle. Cardiovascular Research, 6, 199.

Bellhouse, B. J., and Bellhouse, F. H. (1968a). Thin-film gauges for the measurement of velocity or skin friction in air, water, or blood. Fournal of Scientific Instruments, I (Series 2), I2II.

Bellhouse, B. J., and Bellhouse, F. H. (1968b). Mechanism of closure of the aortic valve. Nature (London), $217,86$.

Bellhouse, B. J., and Bellhouse, F. H. (1969a). Fluid mechanics of model normal and stenosed aortic valves. Circulation Research, 25, 693.

Bellhouse, B. J., and Bellhouse, F. H. (1969b). Fluid mechanics of the mitral valve. Nature (London), 224, 6r5.

Roberts, W. C., and Morrow, A. G. (1967). Secondary left ventricular endocardial fibrosis following mitral valve replacement: a cause of unexplained cardiac failure in the late postoperative period. Circulation, 35, Suppl. II, 224.

Ross, D. N. (1972). Biologic valves; their performance and prospects. Circulation, 45, 1259.

Requests for reprints to A. J. Gunning, F.R.C.S., Nuffield Department of Surgery, Radcliffe Infirmary, Oxford. 